\title{
Comparative Study of Refractive Index Sensing Based on Three Differ- ent Long-Period Grating Configurations by Pulsed ArF Excimer Laser
}

\author{
Jian-Neng Wang ${ }^{* 1}$, Wei-Te Wu², Chien-Hsing Chen ${ }^{3}$ \\ ${ }^{* 1}$ Department of Civil and Construction Engineering, National Yunlin University \\ of Science and Technology, Douliou 64002, Taiwan \\ E-mail: wangjn@yuntech.edu.tw \\ ${ }^{2}$ Department of Biomechatronics Engineering, National Pingtung University \\ of Science and Technology, Pingtung 91201, Taiwan \\ ${ }^{3}$ Department of Physics, National Chung Cheng University, Chiayi 62102, Taiwan
}

\begin{abstract}
Using a pulsed 193-nm 10-Hz ArF excimer laser with the amplitude mask technique, we report a comparative study of sensing capabilities (refractive index and chemical solution) for three different long-period grating configurations written in a Furukawa single mode fiber. The three gratings investigated in this work were a typical long-period grating, a phase-shifted long-period grating, and a long-period grating written in a biconical tapered fiber. The principle of operation is based on the measurement of resonant wavelength shift through the change of refractive index of medium surrounding the cladding surface of the gratings. Previous studies of the three different gratings were conducted by using different fabrication methods (point-to-point writing), irradiation sources (electric-arc discharges, frequency doubled argon ion laser, and $\mathrm{CO}_{2}$ laser) and fiber types. The amplitude mask technique with a 193-nm ArF excimer laser has successfully been produced the three different long-period grating configurations and yielded comparable sensing performance with high sensitivity. Experimental results demonstrated that these fiber grating sensors could provide a resolution of $\sim 10^{-3}$ to $10^{-4}$ for refractive indices in the range of 1.34 to 1.41 , suggesting that these sensors are attractive for chemical sensing and RI measurements with aqueous solutions.

DOI: 10.2961/jlmn.2017.03.0001
\end{abstract}

Keywords: excimer laser, long-period fiber grating, sensor, phase shift, refractive index, concentration, chloride, tapered fiber

\section{Introduction}

The simple, reliable and precise determination of refractive index (RI) of liquid substances is of great important for a variety of applications, such as salinity of water, oil, and food industries. For example, one important application is the prevention of food adulteration for liquids of common use, such as edible oils, wines, and juices. The measurement of optical RI of the substance can be realized in several ways, depending on the type of samples (solids, liquids, etc.) and detection techniques. Various methods of RI measurement have been described in the past years [1-8]. Among them, fiber-optic sensors are one of the best choices, primarily due to their immunity to electromagnetic interference, high temperature resistance, capability of being multiplexed in a large number of independent channels, readily embedded into the composite, ruggedness even in corrosive and other harsh environments, and small size [9-11]. The use of a fiber-optic RI sensor has offered a very powerful tool to perform remote, on-line, and in situ monitoring of many quality-control industrial processes in order to check concentration stability and abnormal aging effects. It is readily shown to work fine for liquids or polymer composites, takes only a small magnitude and can be applied as chemical or biochemical sensors.

For instance, fiber grating sensors [12-15], such as fiber Bragg grating (FBG) or long-period fiber grating (LPG), have been demonstrated to have high sensitivity to the RI of the ambient media and have been widely used to measure the RI of different liquids. In particular, the RI measurement using the LPG sensors [16-20] has attracted great attentions as they can offer a number of favorable features over other optical fiber sensors, such as high sensitivity, large measurement range, ability for multi-parameters measurement, and absolute wavelength modulation which is insensitive to overall system light intensity. They also have some specific characteristics, such as low back-reflection, low insertion loss, polarization independence, and relatively simple of fabrication.

The operation of a LPG is based on the coupling of the fundamental core mode to some forwardpropagating cladding modes of different orders. The coupling of the light into the cladding region results in series of resonant bands centered at certain wavelengths in the transmission spectrum, since a cladding mode is rapidly attenuated in the fiber due to the scattering loss. The core-cladding coupling occurs at a 
specific wavelength $\lambda$ when the phase matching condition is satisfied: $\lambda=\left[\bar{n}_{\text {core }}-\vec{n}_{\text {clad }}\right] \Lambda$, where $\bar{n}_{\text {core }}$, $\vec{n}_{\text {clad }}$, and $\Lambda$ are the effective RI of the fundamental core mode, the effective RI of cladding modes and the period of fiber gratings, respectively. Note that $\bar{n}_{\text {core }}$ is related to RI of the fiber core $n_{\text {core }}$, but $\vec{n}_{\text {clad }}$ depends on both the RI of the cladding $n_{\text {clad }}$ and the surrounding medium $n_{s}$. Those resonant (attenuation) bands at discrete wavelengths are highly sensitive to RI of surrounding. When the concentration or the RI of the surrounding medium changes, $\vec{n}_{\text {clad }}$ changes accordingly and a wavelength shift in the transmission spectrum can be observed. The wavelength shift can be linearly related to the concentrations of the solution under test. For chemical or RI sensing applications, loss peaks from higher order cladding modes are more desirable since they are more sensitive to variations of surrounding RI (SRI) than lower order ones. In addition, LPG can be sensitive to the changes in temperature, strain and bending by fiber. Therefore, temperature changes and deformations must be compensated or prevented in order to correctly measure variations in concentrations.

For a LPG sensor, the spectral sensitivity to RI change of surrounding medium, denoted as $d \lambda / d n_{s}$, arises from the dependence of the resonance wavelength on the effective RI of cladding mode, which is also dependent on the RI of the surrounding materials $n_{s}$. Since both the grating period and the RI of the core mode remain unchanged under the variations of ambient RI $\left(n_{s}\right)$, the spectral RI sensitivity of a LPG can be derived from its phase-matching conditions and expressed by: $d \lambda / d n_{s}=\left(d \lambda / d \vec{n}_{\text {clad }}\right) \times\left(d \vec{n}_{\text {clad }} / d n_{s}\right)$.

It can be seen that the spectral RI sensitivity is expected to have a strong dependence on the order of the cladding mode because for each cladding mode the term $d \vec{n}_{\text {clad }} / d n_{s}$ is different. As ambient RI increases, the spectral sensitivity increases monotonically to a maximum values at a value of $n_{s}$ in which the cladding mode is converted to radiation mode. For a given cladding mode, the wavelength shift $\Delta \lambda$ arising from the ambient RI change may be positive or negative, depending on the characteristic phase-matching curve of $d \lambda / d \Lambda$. The sensitivity to RI change can be enhanced by manipulating the fiber parameters to achieve specific orders of cladding modes, and hence, the effective RI of cladding modes is modified [16]. For example, pure-silica-core fibers with the cladding layer doped with fluorine exhibit a smaller RI than standard communication fibers. Furthermore, the RI sensitivity can also be increased by some post processing of the fiber, such as by etching the fiber cladding [21], by a carbon-nanotube-deposited LPG [22], by zinc oxide coated LPG [23], by using a LPG in a Michelson configuration [24], by cascading two LPGs in a phase-shifted LPG [25], based on Mach-Zenhder interferometer configuration [26], using a photonic crystal fiber interferometer [27], by two concatenated dual-resonance LPGs structure [28], using reflectionbased phase-shifted LPG [29], by writing a LPG in a biconical tapered fiber [30-31], and manipulating biconical tapered fibers for RI measurement $(\mathrm{RI}=$ $1.00 \sim 1.32$ ) [32].

The typical period of a LPG is on the order of a hundred micrometers, which corresponds with an RI modulation variation of the same magnitude of order in the fiber core for fabrication. Compared with the sub-micrometer period of FBGs, the easy fabrication for LPGs offers a practical advantage to apply these devices in sensing applications. LPGs can be made by using ultraviolet (UV) laser irradiation [14, 19, 33], infrared $\mathrm{CO}_{2}$ laser heating [34], electric-arc discharging [35], femtosecond laser radiation [36], and mechanical pressure [37]. The point-to-point writing and amplitude mask technique have offered power tool to produce a desirable pattern of RI modulation on the fiber at each machining position. Several novel LPGbased refractometric configurations can thus be easily exploited, such as phase-shifted LPGs (PS-LPG), Mach-Zenhder or Michelson interferometers based on LPGs. It has been shown by various studies that the resolution of RI for the LPG-based sensors is within the range of $\sim 10^{-3}$ to $10^{-5}$.

In this work, we investigated three types of LPGbased sensors for their sensitivities to surrounding medium's RI for chemical sensing and RI measurements. The three sensors used were a traditional LPG, a PSLPG, and a LPG written in a biconical tapered fiber (BT-LPG). A comparative study of RI sensitivities among these LPG-based sensors shows that they could provide a limiting resolution of $\sim 10^{-3}$ to $10^{-4}$ for refractive indices in the range of 1.34 to 1.41 , suggesting that these sensors could be attractive for use with aqueous solutions for chemical sensing and RI measurement applications.

\section{Experimental}

As described above, several fabrication methods for writing LPGs in optical fibers have been demonstrated and studied. Among them, the use of UV laser irradiation has provided a simple and highly controllable method for photoinducing periodic index changes on a bare optical fiber. The three LPG-based configurations studied in this paper were fabricated in a standard communication single mode fiber (Furukawa SM-332), utilizing an amplitude mask technique and a pulsed 193-nm ArF excimer laser with a laser energy of $~ 135$ $\mathrm{mJ} / \mathrm{cm}^{2}$ and total exposure time of $\sim 3$ minutes operating at $10 \mathrm{~Hz}$. The Furukawa optical fiber SM-332 is a matched cladding design single mode fiber made of synthesized silica with excellent low bending loss for the fabrication of low-loss fused taper and fiber sensors. It is a $245 \pm 10 \mu \mathrm{m}$ coating diameter fiber consisting of a germanium-doped core surrounding a silica cladding with a diameter about $125 \pm 1 \mu \mathrm{m}$. The nominal mode field diameter is 9.5 11.5 $\mu \mathrm{m}$ at $1550 \mathrm{~nm}$ and the index of refraction difference between core and cladding is $0.33 \%$. Shown in Figure 1 is the ex- 
perimental setup for LPG fabrication. A broadband EELED fiber source and a high-resolution spectrum analyzer (ANDO AQ6315A) were used to in-situ monitor the transmission loss. Photosensitivity enhancements, such as hydrogen loading and flame brushing, were widely applied to improve the weak laser interaction of standard telecom fiber. The fibers were hydrogen loaded at a pressure of 120 bars over a period of two weeks at room temperature. The length of three types of LPG-based sensors was around $2 \sim 3 \mathrm{~cm}$, and the grating period was set to $\sim 380 \mu \mathrm{m}$.

Fabrication of a PS-LPG fiber followed the same procedure to that of a normal LPG, in which a section of LPG was inscribed in an optical fiber and then another LPG was made on the same fiber with a designed phase shift to the first one. A home-made amplitude mask (Figure 2) was produced by using a $\mathrm{CO}_{2}$ laser pulse for this purpose. The phase of PS-LPGs used through this study was $\pi / 2$. Figure 2(a) displays the schematic illustration of a PS-LPG, where $\Delta L$ is the distance between two LPGs and phase shift is $\theta=2 \pi \Delta L / \Lambda$, and Figure 2(b) shows the photography of a home-made amplitude mask for the fabrication of this PS-LPG. Figure 3 is a microscopic photography of a home-made phase-shifted amplitude mask on the top of a bar fiber during the fabrication. Most of the techniques (such as electric-arc discharges and point-topoint writing on a step-index single mode fiber) to produce the PS-LPG reported by number of other authors were different from ours (ArF excimer laser and amplitude mask writing on a Furukawa single mode fiber) and it would be interesting to examine and compare its sensing sensitivity between different approaches.

The technique to fabricate a BT-LPG was based on that used by T. Allsop et al. in Ref. 30, in which the BT-LPG was fabricated with the point-to-point technique using a frequency doubled argon ion laser. In principle, the design of a BT-LPG itself was similar to a Mach-Zehnder LPG interferometer, but can reduce the problems associated with the conventional fiber Mach-Zehnder LPG. Previous study has shown that for this type of LPG, the RI resolution is around $10^{-4}$ for aqueous solutions. The biconical tapers (see Figure 4) were fabricated by applying to a flame with a controlled tension to the fibers. The total length of tapered fibers was around $3 \mathrm{~cm}$ with an approximate minimum fiber radius of $34 \pm 2 \mu \mathrm{m}$. LPGs were then inscribed into the tapered fiber by using a pulsed 193-nm ArF excimer laser with an amplitude mask technique. The fabrication method (amplitude mask writing), irradiation source (ArF excimer laser) and fiber type (Furukawa SM-332 single mode fiber) used in this study were different from the previous work by T. Allsop et al. [30-31]. After the laser exposure, all the LPGs were annealed at $150{ }^{\circ} \mathrm{C}$ for 24 hours to stabilize their optical properties.

A typical transmission spectrum of a PS-LPG is shown in Figure 5. With suitable fabrication parameters, such as laser power, exposure time, phase-shift, width and length of the tapered fiber, and grating peri- od, the resulting resonance wavelengths ranging from $1100 \mathrm{~nm}$ to $1600 \mathrm{~nm}$ with a greater than $15 \mathrm{~dB}$ peak depth were obtained. In this work, a number of these three types of LPG-based sensors associated with various attenuation bands (corresponding to various cladding modes) were measured and analyzed.

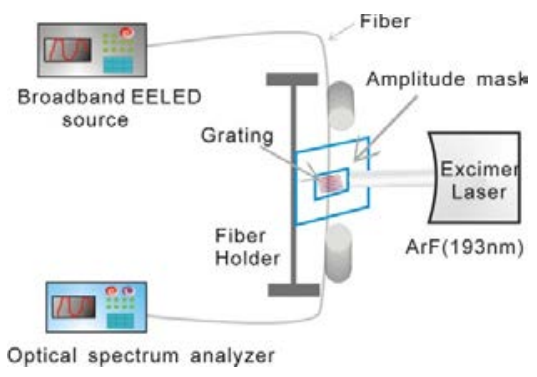

Fig. 1 Schematic of experimental setup.

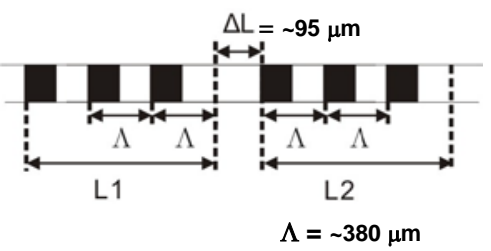

(a)

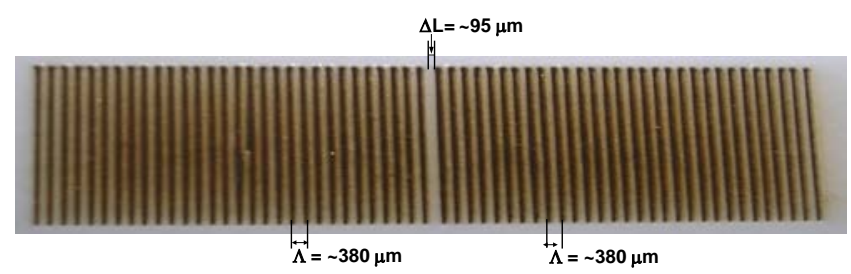

(b)

Fig. 2 A home-made amplitude mask for the fabrication of a PS-LPG fiber. (a): Schematic illustration of a PSLPG, where $\Delta L$ is the distance between two LPGs and phase shift ; (b): Photography of a home-made amplitude mask for the fabrication of a PS-LPG sample of the type of graph you could make.

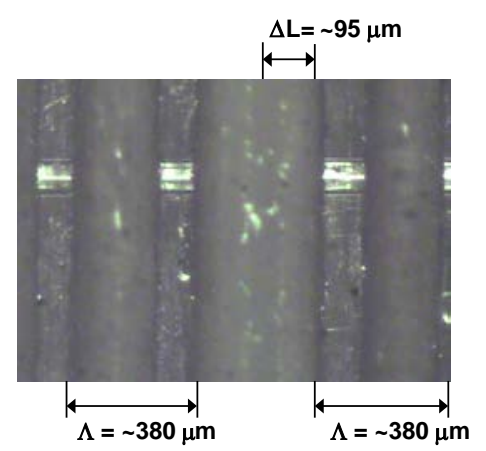

Fig. 3 Microscopic photography of a home-made phaseshifted amplitude mask on the top of a bar fiber. 


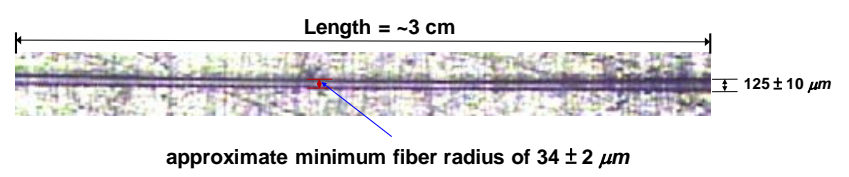

Fig. 4 Microscopic photography of a section of a biconical tapered fiber.

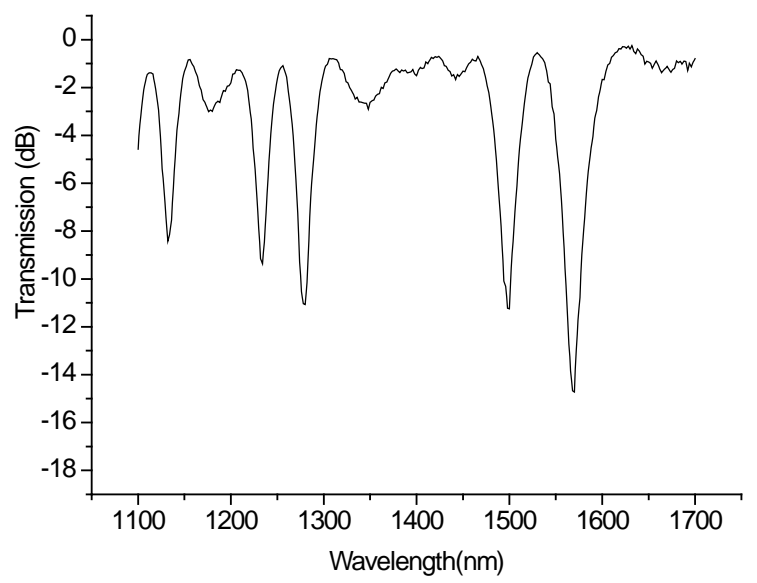

Fig. 5 A typical transmission spectrum of a PS-LPG.

\section{Results and discussion}

The characteristics of all fabricated LPGs, such as temperature and strain, were first investigated. The thermal responses of the LPGs were measured by heating the grating from $30{ }^{\circ} \mathrm{C}$ to $150{ }^{\circ} \mathrm{C}$ in an incremental step of $15{ }^{\circ} \mathrm{C}$, using a temperature-controllable chamber. When temperature was increased, the resonance wavelength of LPGs was shifted to the longer or the shorter wavelength, showing that temperature sensitivity ( $d \lambda / d T$ ) of LPGs exhibited either positive or negative sensitivity. The slope of a linear fit of measured wavelength shifts to surrounding temperature changes was determined as the temperature sensitivity of the investigated grating sensor. The temperature sensitivities for the investigated LPG, PS-LPG, and BT-LPG were determined to be around 0.06-0.09 $\mathrm{nm}^{\circ} \mathrm{C}^{-1}, 0.04-0.09 \mathrm{~nm}^{\circ} \mathrm{C}^{-1}$, and $0.07-0.08 \mathrm{~nm}^{\circ} \mathrm{C}^{-1}$, respectively, which were several times larger than those of FBGs $\left(\sim 0.01 \mathrm{~nm}^{0} \mathrm{C}^{-1}\right)$. It could be seen that all three LPG-based sensors possessed almost similar responses in magnitude to temperature changes.

The spectral sensitivities to the strain of the LPG were performed by mounting the grating on a translation stage that was moved outward to induce a fixed magnitude of strain in the optical fiber grating. The slope of a linear fit of measured wavelength shifts to applied strain changes was determined as the strain sensitivity of the investigated grating sensor. For all LPGs used in this work, the strain sensitivities at various resonance wavelengths were found to be very small $(\sim 0.09 \pm 0.01 \mathrm{pm} / \mu \varepsilon)$ and exhibited nearly insensitive to strain variations.
In order to characterize the LPG as an RI sensor or a chemical concentration sensor, measurements with sucrose and sodium chloride $(\mathrm{NaCl})$ aqueous solutions were performed. The SRI was controlled through the use of sucrose solutions with various concentrations [38-39]. The ability of three LPG-based sensors to detect changes in the SRI or chemical solution concentrations was then studied. The experiment used to measure RI sensitivity was based on Ref. 39, which included a broadband light source, a sensing LPG fiber, a cell, and an optical spectrum analyzer. The LPG sensor was immersed in different media, such as air, water and sucrose solutions, and its transmission spectrum was measured with the optical spectrum analyzer. Each transmission spectrum was referenced to the background spectrum of a bare fiber in air. For RI sensitivity measurements, the LPG was immersed in a sucrose solution, while for chemical sensing measurements, the LPG was immersed in a $\mathrm{NaCl}$ solution. The LPG was carefully cleaned, first washed in methanol, then in deionized water and finally dried before it was immersed into the next test solution. The sensing LPG fiber was applied by a small and fixed magnitude of tension to minimize bending the fiber. Therefore, the results reported here were not influenced by temperature, strain and bending effects.

When the concentration and, hence, the RI of a sucrose solution increased in the range of 1.34-1.41, the transmission spectrum of the LPG sensor exhibited either a linear decrease (LPG and PS-LPG) or a linear increase (BT-LPG) in the peak wavelength (resonance wavelength). Regarding the resonance wavelength for each transmission spectrum, the 3- $\mathrm{dB}$ bandwidth method was used to calculate the resonance wavelength [40]. For example, Figure 6 shows wavelength shifts of the typical LPG, PS-LPG, and BT-LPG sensors against sucrose concentration and, hence, the corresponding changes in SRI. A linear regression approach was employed to calculate the RI sensitivity of each type of LPG sensor [39, 41]. The slope of a linear fit of measured wavelength shifts to SRI changes was determined as the RI sensitivity of the investigated LPG grating sensor. Figure 7 presents a linear fit to the plot of peak wavelength as a function of RI for the typical LPG, PS-LPG, and BT-LPG sensors, respectively.

First, the results display that both Figure 7(a-b) exhibits blue shifts in wavelength; however, Figure 7(c) shows red shifts in wavelength. As RI increased, the wavelength shifts decreased for both the typical LPG and PS-LPG sensors; though the wavelength shifts increased BT-LPG sensors. For a given cladding mode, the wavelength shift $\Delta \lambda$ arising from the ambient RI change may be positive or negative, depending on the characteristic phase-matching curve of $d \lambda / d \Lambda$. Thus, the sensitivity to RI change can be enhanced by manipulating the fiber parameters or configurations to achieve specific orders of cladding modes, and hence, the effective RI of cladding modes is modified [16]. T. Allsop et al. have reported that two tapered LPG fiber sensors with a period of $350 \mu \mathrm{m}$ and $400 \mu \mathrm{m}$, respec- 
tively, exhibit a spectral feature that splits and separates (bifurcation effect); thus, both two tapered LPG sensors possess either a blue or red wavelength shift as a function or RI [31].

Second, our present results show that RI sensitivities of the LPG, PS-LPG, and BT-LPG were -59.9 $\mathrm{nm} / \mathrm{RI},-81.3 \mathrm{~nm} / \mathrm{RI}$, and $+271.7 \mathrm{~nm} / \mathrm{RI}$, respectively, which led to a limit of detection (LOD $=3 \sigma / \mathrm{m}, \sigma=$ standard deviation of transmission loss peak in measuring the blank sample, $m=$ slope) in index, $1.0 \times 10^{-3}$, $6.9 \times 10^{-4}$, and $\sim 2 \times 10^{-4}$, respectively. The investigation shows that BT-LPG exhibited the highest sensitivity to SRI among three LPG types of sensors.

The sensitivity of LPG to concentration change in chemical solution was further investigated using sodium chloride aqueous solutions. Sodium chloride, also known as common salt or halite, is one of the most important naturally occurring substances that is most responsible for the salinity of the ocean. The detection of chloride ion concentration is important in a variety of industrial applications, including pure, environmental, bio- and industrial chemistry. In particular, the salt damage of concrete structure due to the corrosion of reinforced bars in marine environment is normally caused by the penetration of chloride ion into concrete. The LPG under test was immersed in a container of deionized water (100 ml volume) and increments of 5 $\mathrm{ml}$ of 4 molar $\mathrm{NaCl}$ solutions were added. Figure 8 shows wavelength shifts of a typical PS-LPG against molar concentration of $\mathrm{NaCl}$, and hence the corresponding changes in SRI. Figure 9 shows a linear fit (correlation coefficient, $\mathrm{R}^{2}=0.9423$ ) to the plot of peak wavelength for a typical PS-LPG as a function of molar concentration of $\mathrm{NaCl}$. Similar analyses were applied for LPG and BT-LPG grating sensors to extract their sensitivities to concentration change in $\mathrm{NaCl}$ solution. Our results show that sensitivities of LPG, PS-LPG, and BT-LPF in terms of $\mathrm{NaCl}$ molar concentration were $-0.30 \mathrm{~nm} / \mathrm{M},-0.70 \mathrm{~nm} / \mathrm{M}$, and $0.57 \mathrm{~nm} / \mathrm{M}$, respectively, leading to a limit of detection in molar concentration, $0.2,0.08$, and 0.1 , respectively. The limit of detection presented here could be easily enhanced if a spectrum analyzer with spectral resolution of $0.1 \mathrm{pm}$ is employed.

The BT-LPG showed the highest RI sensitivity of the three grating sensors, while shows sensitivity to $\mathrm{NaCl}$ concentration that was lower or equal to the other two grating sensors. If we assume that the BT-LPG responds only to RI, then its SRI sensitivity is reduced by a factor of three in aqueous $\mathrm{NaCl}$ as compared to that in sucrose solutions. It might imply that the BTLPG offers selectivity in its sensitivity, and was thus not measuring only RI. Since the $\mathrm{NaCl}$ solutions were solutions with RIs close to the pure water's RI, perhaps only in that region of refractive indices (close to 1.33) the PS-LPGs were more sensitive. That less sensitivity of BT-LPG to $\mathrm{NaCl}$ molar concentration was interesting but still remains to be clarified and the investigation is underway. However, studies presented here demonstrate the feasibility of using the design of
LPG-based sensor for RI measurement or chemical sensing with high sensitivity.

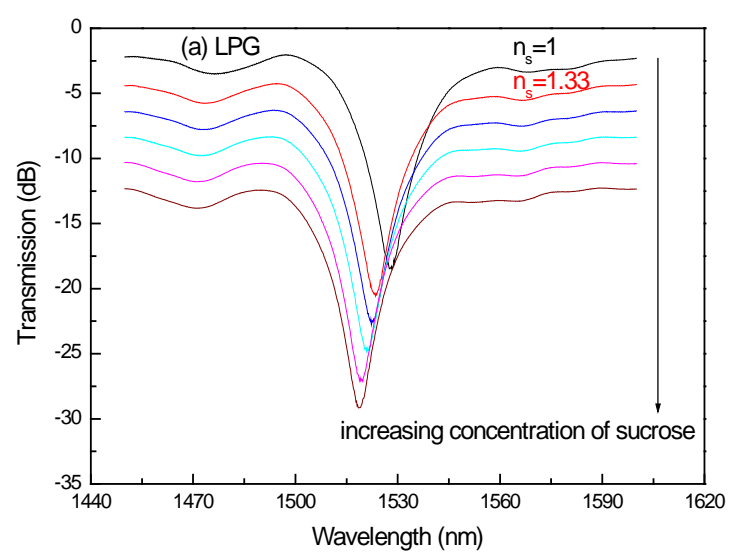

(a)

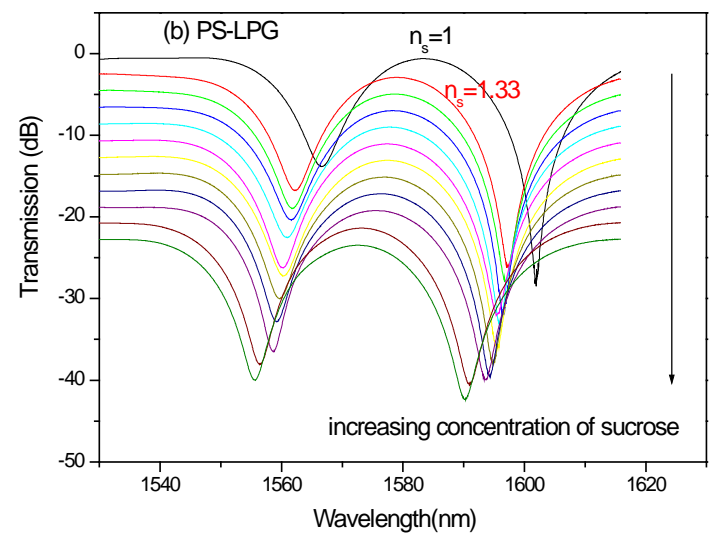

(b)

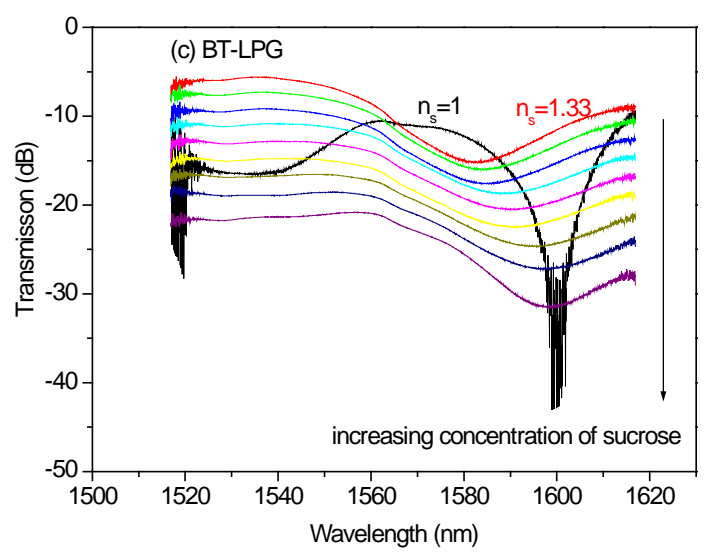

(c)

Fig. 6 Transmission spectra of a typical (a): LPG; (b): PS-LPG; and (c): BT-LPG sensors in aqueous solution containing increasing concentration of sucrose (from top to bottom). 


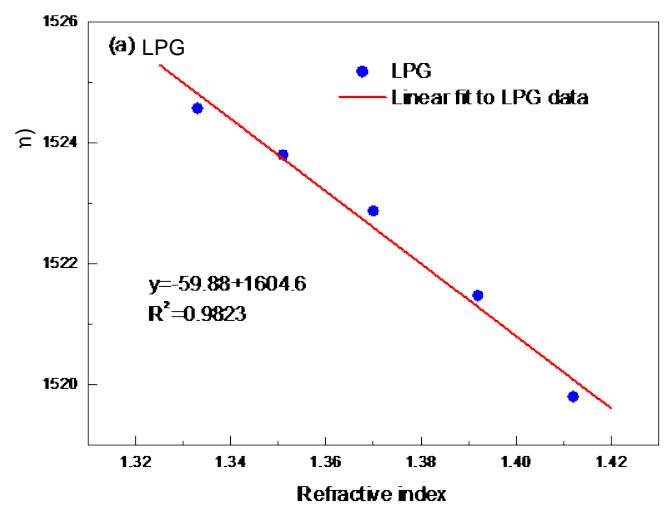

(a)

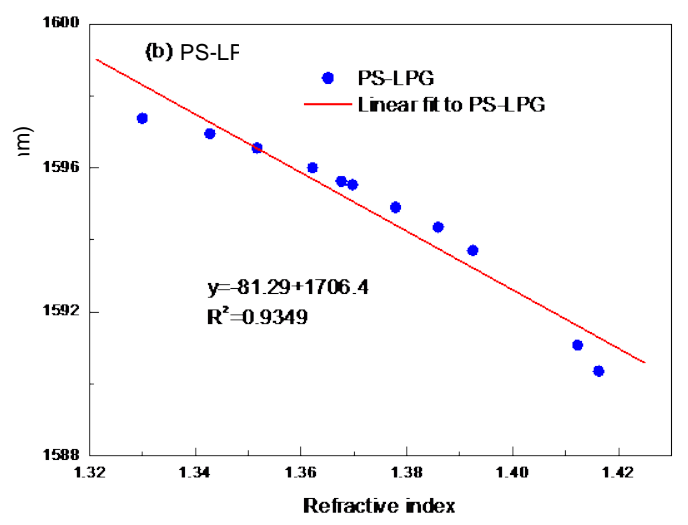

(b)

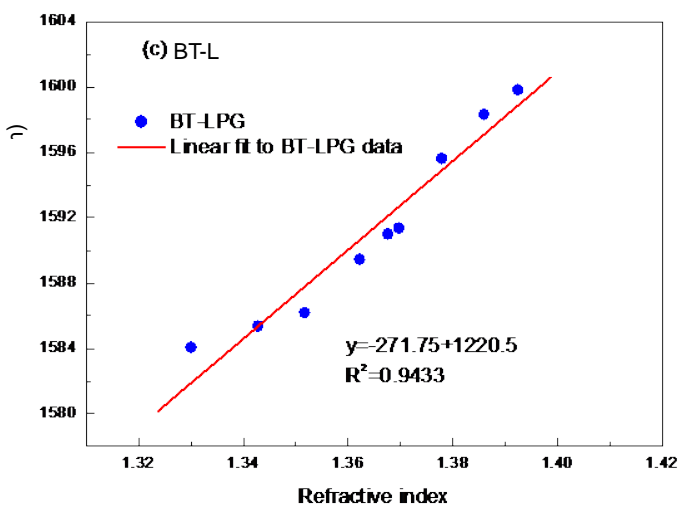

(c)

Fig. 7 Plots of peak wavelength versus RI of the sucrose solution for a typical (a): LPG; (b): PS-LPG; and (c): BT-LPG sensors in aqueous solution containing increasing concentration of sucrose.

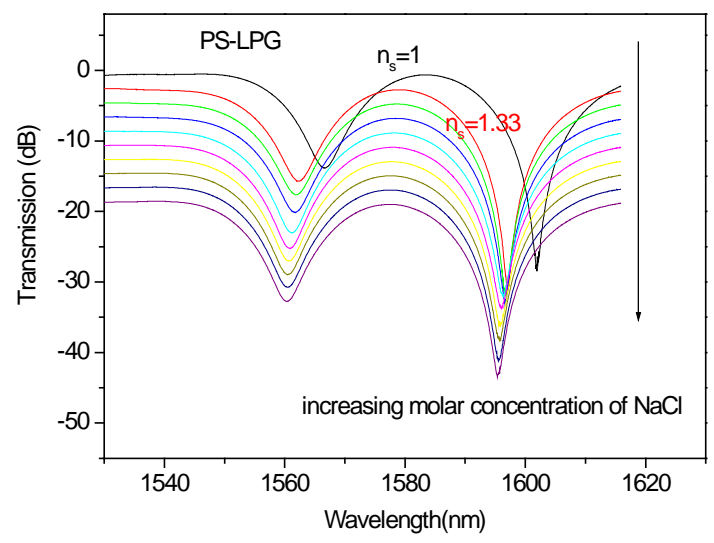

Fig. 8 Transmission spectra of a typical PS-LPG sensor in aqueous solution containing increasing concentration of sodium chloride (from top to bottom).

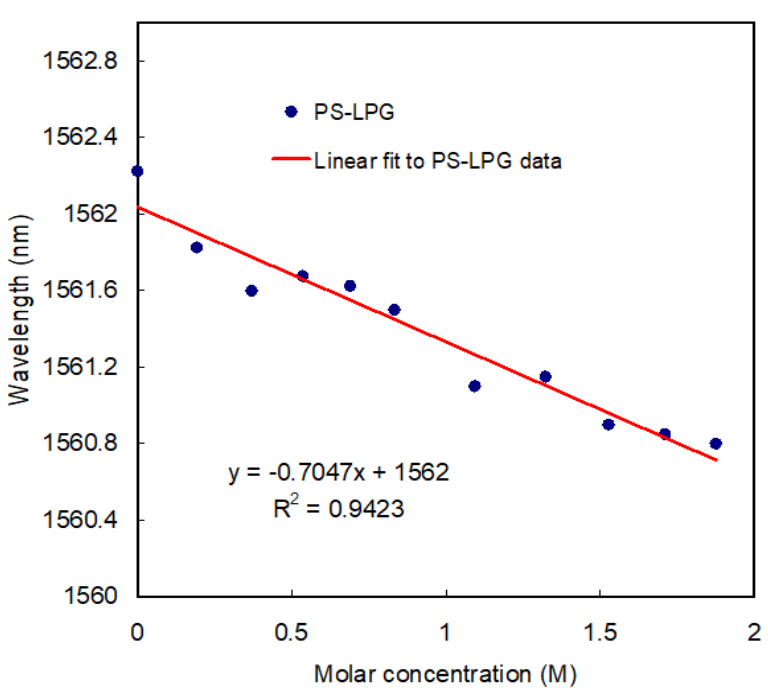

Fig. 9 Plot of peak wavelength of a typical PS-LPG versus molar concentration of sodium chloride solutions.

\section{Conclusions}

Using a 193-nm 10-Hz ArF excimer pulse laser associated with the amplitude mask technique, we have successfully fabricated and demonstrated three different LPG configurations (LPG, PS-LPG, and BT-LPG) in a Furukawa SM-332 single mode fiber. Previous studies of the three LPG grating sensors were performed by using different fabrication methods (pointto-point writing), irradiation sources (electric-arc discharge, frequency doubled argon ion laser, 157-nm F2Laser, $\mathrm{CO}_{2}$ laser) and types of optical fiber (Corning SMF-28, Fiber core single mode fiber, SMPS 1300$125 \mathrm{P})$. We report on a comparative study of sensing capabilities (RI, and chemical solution) based on these three different LPG configurations. It is interesting to examine and compare their physical sensing sensitivities with different fabrication approaches. Our results 
demonstrate that LPG-based sensors could provide a limiting resolution of $\sim 10^{-3}$ to $10^{-4}$ for refractive indices in the range of 1.34 to 1.41 , suggesting that these sensors may be suitable for use with aqueous solutions for chemical sensing and RI measurement. The potential for LPG-based sensors to provide a sensitive measurement of concentration in solution was also shown. It is interesting to learn that our data show comparable and similar results of RI sensitivity from most previous studies but yield a slight different trend with the $\mathrm{NaCl}$ solutions. In the sucrose concentrations, it seemed that the PS-LPGs were less sensitive than the BT-LPGs, but with the $\mathrm{NaCl}$ solutions it was just the opposite. It might hint that the BT-LPGs provided selectivity in its sensitivity to surrounding $\mathrm{RI}$ in $\mathrm{NaCl}$ solutions, in which the region of refractive indices was close to pure water. Further work to clarify this remark is underway.

Studies presented in this paper illustrate the feasibility of making three different LPG configurations using the ArF excimer laser pulses in the same type of single mode fiber for chemical and biochemical sensing. The realization of the sensors was through the measurement of transmission spectra of the sensing fiber grating. The advantage of this type of the sensor is relatively simple of construction, small, light, robust, low-cost, and ease of use. Moreover, the sensors possess the potential capability for on-site, in vivo, and remote sensing, and have the potential use for disposable sensors. Furthermore, by modifying the grating surface with a monolayer of colloidal gold or silver nanoparticles, the sensors could be further developed to perform label-free detection for the diagnosis of diseases, food, and environmental detection of biological agents to satisfy needs of emerging chemical and biological detection technologies. The linearity and sensitivity of the sensors are also useful for biosensor applications but have not yet been optimized, which could be enhanced by optimizing some key parameters, such as fabrication methods (point-to-point writing), irradiation sources (electric-arc discharges, $\mathrm{CO}_{2}$ laser, and femtosecond laser pulses), length and period of the fiber gratings, phase shift of the PS-LPG, width and length of the tapered fibers, and the fiber types.

\section{Acknowledgements}

This work was partially supported by the by the Ministry of Science and Technology (Taiwan) under Contract MOST Nos. 104-2811-M-194-004, 1042914-I-007-A1, 105-2221-E-224-014, and 106-2221E-224-017 is acknowledged. The authors thank Prof. Dr. Jaw-Luen Tang for his substantial contribution to this study especially on the conception and data acquisition.

\section{References}

[1]. G.F. Stanley: Refractometers: Basic Principles (1989).

[2]. J. Rheims, J. Koser and T. Wriedt: Meas. Sci. Technol. 8 (1997) 601.

[3]. R.C. Peter: Appl. Opt. 22 (1983) 3070.
[4]. K. Schmid and A. Penzkofer: Appl. Opt. 22 (1983) 1824.

[5]. N. Shojiro: Appl. Opt. 31 (1992) 6690.

[6]. R. Aschauer, A. Asenbaum, C. Vasi and E. Wilhelm: Meas. Sci. Technol. 11 (2000) 1714.

[7]. Y. Hiroshi: J. Phys. E. 21 (1988) 571.

[8]. R.S. Madhusudana: Opt. Eng. 36 (1997) 162.

[9]. E. Udd: Fiber Optic Sensors: An Introduction for Engineers and Scientists (1991).

[10]. J. Dakin and B. Culshaw: Optical Fiber Sensors: Principals and Components vol. 1 (1988).

[11]. B. Culshaw and J. Dakin: Optical Fiber Sensors: Systems and Applications vol. 2 (1989).

[12]. R. Kashyap: Fibre Bragg Gratings (1999).

[13]. A.D. Kersey, M.A. Davis, H.J. Patrick, M. LeBlanc, K.P. Koo, C.G. Askins, M.A. Putnam and E.J. Friebele: J. Lightwave Technol. 15 (1997) 1442.

[14]. V. Bhatia and A.M. Vengsarkar: Opt. Lett. 21 (1996) 692.

[15]. L. Zhang, W. Zhang, and I. Bennion: "In-fiber grating optic sensors." Fiber Optic Sensors (2002) 123

[16]. H.J. Patrick, A.D. Kersey and F. Bucholtz: J. Lightwave Technol. 16 (1998) 1606.

[17]. R. Falciai, A.G. Mignani and A. Vannini: Sensors Actuat. B: Chem. 74 (2001) 74.

[18]. M.N. Ng, Z.H. Chen and K.S. Chiang: IEEE Photon. Technol. Lett. 14 (2002) 361.

[19]. S. James and R. Tatam: Meas. Sci. Technol. 14 (2003) R49.

[20]. J.H. Chong, P. Shum, H. Haryono, A. Yohana, M.K. Rao, C. Lu and Y. Zhu: Opt. Commun. 229 (2004) 65.

[21]. K.S. Chiang, Y. Liu, M.N. Ng and X. Dong: Electron. Lett. 36 (2000) 966.

[22]. Y.C. Tan, W.B. Ji, V. Mamidala, K.K Chow, and S. C Tjin: Sensors Actuat. B: Chem. 196 (2014) 260.

[23]. L. Coelho, D. Viegas, J.L. Santos, and J.M.M.M De Almeida: Sensors Actuat. B: Chem. 223 (2016) 45.

[24]. P.L. Swart: Meas. Sci. Technol. 15 (2004)157680.

[25]. R. Falate, O. Fraza, G. Rego, J.L. Fabris and J.L. Santos: Appl. Opt. 45 (2006) 5066-72.

[26]. B.A.L. Gwandu, X. Shu, T.D.P Allsop, W. Zhang, L. Zhang and I. Bennion: Electron. Lett. 38 (2002) 695-6.

[27]. J.N. Wang and J.L. Tang: Sensors 12 (2012) 2983.

[28]. S.M. Tripathi, W.J. Bock, A. Kumar, and P. Mikulic: Opt. let. 38 (2013): 1666.

[29]. J. Huang, X. Lan, A. Kaur, H. Wang, L. Yuan, and H. Xiao: Opt. Eng. 52 (2013): 014404.

[30]. T. Allsop, F. Floreani, K. Jedrzejewski, P.V.S. Marques, R. Romero, D.J. Webb and I. Bennion: Electron. Lett. 41 (2005) 8.

[31]. T. Allsop, F. Floreani, K. Jedrzejewski, P. Marques, R. Romero, D. Webb and I. Bennion: J. Lightwave Technol. 24 (2006) 870-8. 
[32]. S. Mas, J. Martí, and J. Palací: IEEE Sensors J. 15 (2015) 1331.

[33]. K.P. Chen, P.R. Herman and J. Zhang: Opt. Lett. 26 (2001) 771-3.

[34]. D.D. Davis, T.K. Gaylod, E.N. Glytsis, S.G. Kosinski, S.C. Mettler and A.M. Vengsarkar: Electron. Lett. 34 (1998) 302-3.

[35]. G. Rego, O. Okhotnikov, E. Dianov and V. Sulimov: J. Lightwave Technol. 19 (2001) 1574-9

[36]. Y. Kondo, K. Nouchi, T. Mitsuyu, M. Watanabe, P.G. Kazansky and K. Hirao: Opt. Lett. 24 (1999) 646-8.

[37]. S. Savin, M.J.K. Digonnet, G.S. Kino and H.J. Shaw: Opt. Lett. 25 (2000) 710-2.

[38]. T.C. Hwang: Experiments in Physical Chemistry (Taipei: Gaulih, 1994). (Book in Traditional Chinese)

[39]. S.F. Cheng and L.K. Chau: Anal. Chem. 75 (2003) 16-21.

[40]. J.N. Wang, and C.Y. Luo: Sensors 12 (2012): 4578.

[41]. J.L. Tang, S.F. Cheng, W.T. Hsu, T.Y. Chiang and L.K. Chau: Sensors Actuat. B: Chem. 119 (2006) 105-9.

(Received: June 5, 2017, Accepted: September 24, 2017) 